\title{
EDITORIAL
}

\section{Impact of inequalities in health care on the mortality risk of individuals with severe mental illnesses}

\author{
Elisa Brietzke, ${ }^{1,2}$ Rodrigo B. Mansur, ${ }^{2}$ Roger S. Mclntyre ${ }^{2,3}$ \\ ${ }^{1}$ Research Group of Molecular and Behavioral Neuroscience of Bipolar Disorder, Departamento de Psiquiatria, Universidade Federal de São \\ Paulo (UNIFESP), São Paulo, Brazil. ${ }^{2}$ Mood Disorders Psychopharmacology Unit, University Health Network, Toronto, Canada. ${ }^{3}$ Brain and \\ Cognition Research Foundation, Toronto, Canada.
}

It is well known that individuals with severe mental illnesses (SMI), such as bipolar disorder (BD), major depressive disorder (MDD), and schizophrenia, have a twofold to threefold-higher risk of mortality relative to the general population and a markedly reduced life expectancy (by 15 years on average). Although suicide is unquestionably an important factor, replicated evidence indicates that the main driver of excessive and premature mortality in this population is cardiovascular disease. The burden of cardiovascular disease is evident also in younger age groups, in which heart disease, alongside other physical illnesses, accounts for most of the excess mortality.

A major success story in medicine and public health has been the progressive increase in life expectancy throughout the world. Unfortunately, individuals with SMI do not seem to be benefitting from this trend. Whereas overall mortality rates have decreased sharply, including from disparate conditions such as heart disease, cancer, and infectious diseases, this decline is less evident in the SMI population. For example, the incidence of myocardial infarction has decreased by almost $50 \%$ in the general population, but has barely changed in individuals with SMI.

Several factors have been explored as possible mediators of cardiovascular disease and excess or premature mortality in SMI. These factors include, but are not limited to, metabolic and cardiac effects of medications (especially second-generation antipsychotics, antidepressants and mood stabilizers); poor health habits (e.g., smoking and alcohol abuse); and pathophysiological mechanisms intrinsic to the systemic manifestations of mental disorders. ${ }^{1,2}$ Nevertheless, inequalities in the access to health care and in the quality of its delivery, especially regarding detection and treatment of cardiovascular conditions and other physical problems in people with SMI, have been relatively less discussed.

The evidence is as clear as it is damning. Health systems worldwide, including those in high-income countries, are failing to provide optimal, evidence-based, cost-effective interventions to this already vulnerable population. There is replicated evidence that individuals with SMI are less likely

Correspondence: Elisa Brietzke, 399 Bathrust Street, 9th Floor Main Pavilion, M5TT 2S8, Toronto, ON, Canada.

E-mail: elisabrietzke@ hotmail.com to have a regular source of primary medical care, even after accounting for possible confounders such as age, gender, race, education, income, and health insurance status. ${ }^{3}$ Neither psychiatrists nor general practitioners screen and monitor individuals with SMI appropriately for metabolic risk factors, despite recommendations from several agencies, such as the U.S. Food and Drug Administration, American Diabetes Association, and American Psychiatric Association. For example, the rates of baseline metabolic assessment in individuals taking second-generation antipsychotic range from 8 to $30 \%$, and only $8.8 \%$ of these patients undergo metabolic monitoring as recommended. Individuals with SMI are less likely to be prescribed cardiovascular medications (e.g., antihypertensives, antihyperlipidemics) and to receive smoking cessation advice, even though they are twice as likely to smoke and to have obesity or diabetes. Incredibly, individuals with SMI and a diagnosed myocardial infarction have lower rates of surgical interventions, such as revascularization, stenting, and bypass grafting, and are less likely to receive cerebrovascular arteriography or warfarin following a stroke. As a result, their mortality rate after a myocardial infarction is three times higher than that of the general population. ${ }^{4}$ In addition, people with SMI are less likely to receive optimal clinical care for diabetes and arthritis, and are less likely to receive routine cancer screening. ${ }^{5}$ This pattern of poor access to quality health care includes acute conditions as well, such as acute appendicitis: people with SMI have been found to be diagnosed later, experience more complications (e.g., gangrene or perforation of the appendix), and have worse outcomes after surgery. ${ }^{6}$

Fortunately, evidence indicates that a number of simple and cost-effective interventions can be used to address these issues. Medical facilities providing integrated care, which is sometimes as straightforward as having a psychiatrist and a general practitioner on-site, have been shown to yield better health outcomes. Preventive interventions, including but not limited to patient education, smoking cessation, and weight-loss programs, have been shown to work in the SMI population. Side effects of psychotropic medications can also be managed effectively through a combination of behavioral (e.g., dietary and exercise counseling) and pharmacological interventions (e.g., metformin, liraglutide).

One of the pressing issues of the 21st century is the increase in income inequality over the past decades 
(or, in low- to middle-income countries, its persistence). Recent epidemiological evidence suggests that the aforementioned increase in life expectancy in the general population may not be equally distributed, with lower income/education cohorts displaying lower increases or even decreases in life expectancy. Wealth, socioeconomic status, access to healthcare, mental illness, and health outcomes are all inextricably linked. The extent to which this larger context of social, economic, and cultural trends is affecting the issue of excess/premature mortality in SMI, as well as the extent to which these highly prevalent and chronic SMIs may actually be driving the overall mortality gap, are relevant questions that deserve to be explored with empirical data. At a minimum, societal changes should inform how the issue of excess/premature mortality in SMI is framed, understood, and addressed, as advances in science and technology are apparently benefiting only the mentally healthy part of the population. ${ }^{7} \mathrm{~A}$ commitment to first recognizing and then addressing the problem of excess/premature mortality in SMI is needed from all actors, from healthcare policy makers through healthcare providers to patient and peer advocacy groups.

\section{Disclosure}

The authors report no conflicts of interest.

\section{References}

1 Czepielewski L, Daruy Filho L, Brietzke E, Grassi-Oliveira R. Bipolar disorder and metabolic syndrome: a systematic review. Rev Bras Psiquiatr. 2013;35:88-93.

2 Mansur RB, Brietzke E, McIntyre RS. Is there a "metabolic-mood syndrome"? A review of the relationship between obesity and mood disorders. Neurosci Biobehav Rev. 2015;52:89-104.

3 Bradford DW, Kim MM, Braxton LE, Marx CE, Butterfield M, Elbogen EB. Access to medical care among persons with psychotic and major affective disorders. Psychiatr Serv. 2008;59:847-52.

4 Bodén R, Molin E, Jernberg T, Kieler H, Lindahl B, Sundström J. Higher mortality after myocardial infarction in patients with severe mental illness: a nationwide cohort study. J Intern Med. 2015;277:727-36.

5 James M, Thomas M, Frolov L, Riano NS, Vittinghoff E, Schillinger D, et al. Rates of cervical cancer screening among women with severe mental illness in the public health system. Psychiatr Serv.; 2017 Apr 17: appips201600293. doi:10.1176/appi.ps.201600293. [Epub ahead of print]

6 Cooke BK, Magas LT, Virgo KS, Feinberg B, Adityanjee A, Johnson FE. Appendectomy for appendicitis in patients with schizophrenia. Am J Surg. 2007;193:41-8.

7 Mclntyre RS, Lee Y, Mansur RB. Treating to target in major depressive disorder: response to remission to functional recovery. CNS Spectr. 2015;20:20-30. 